\title{
Examining the Relationship Between Parent and Child Psychopathology in Treatment-Seeking Veterans
}

\author{
Alyson K. Zalta ${ }^{1,2}$ (D) Eric Bui ${ }^{3,4} \cdot$ Niranjan S. Karnik $^{1} \cdot$ Philip Held $^{1} \cdot$ \\ Lauren M. Laifer ${ }^{3} \cdot$ Julia C. Sager $^{3}$ - Denise Zou ${ }^{1}$ - Paula K. Rauch ${ }^{3,4}$. \\ Naomi M. Simon ${ }^{3,4} \cdot$ Mark H. Pollack $^{1} \cdot$ Bonnie Ohye $^{3,4}$
}

Published online: 28 June 2017

(c) The Author(s) 2017. This article is an open access publication

\begin{abstract}
This study aimed to examine: (1) the relationship between parental psychopathology and child psychopathology in military families and (2) parenting sense of competence as a mediator of the relationship between veteran psychopathology and child psychopathology. As part of their standard clinical evaluations, 215 treatmentseeking veterans who reported having a child between the ages of 4 and 17 were assessed for psychopathology (posttraumatic stress disorder, depression, anxiety, and stress), their sense of competence as a parent, and their child's psychopathology (internalizing, externalizing, and attentional symptoms). A path analysis model examining parenting sense of competence as a mediator of the relationship between veteran psychopathology and child psychopathology showed significant indirect effects of veteran depression on all child psychopathology outcomes via parenting sense of competence. Parental sense of competence may be a critical mechanism linking veteran depression and child psychopathology, and may therefore be an important target for intervention.
\end{abstract}

Alyson K. Zalta

Alyson_Zalta@rush.edu

1 Road Home Program, Department of Psychiatry, Rush University Medical Center, 1645 W. Jackson Blvd, Suite 602, Chicago, IL 60612, USA

2 Department of Behavioral Sciences, Rush University Medical Center, Chicago, IL, USA

3 Home Base, a Red Sox Foundation and Massachusetts General Hospital program, Boston, MA, USA

4 Department of Psychiatry, Harvard Medical School, Boston, MA, USA
Keywords Veteran $\cdot$ Military $\cdot$ Parenting $\cdot$ Child psychopathology $\cdot$ Parenting sense of competence

\section{Introduction}

Since September 11, 2001, over 2.6 million US service members have deployed to Iraq and/or Afghanistan, and this number is projected to reach 3.5 million by 2019 [1]. Operation Enduring Freedom, Operation Iraqi Freedom, and Operation New Dawn are fundamentally unique from previous wars and are characterized by repeated and extended deployments, with early evidence suggesting disproportionately high rates of mental health disorders compared to the general US population [2]. The post-9/11 conflicts tax not only military personnel, but also their immediate family members, including 2 million militaryconnected children, hundreds of thousands of whom have experienced at least one parental deployment [3]. Both deployment and reintegration are accompanied by unique stressors for military families, which may be particularly salient for dependent children. These include renegotiation of family roles, responsibilities, and boundaries; changes in routines; lack of awareness about military service within communities; and media exposure that may present incomplete information or negative aspects of deployment [4-6].

While children generally adapt well to mild and shortlived stressors [7], sustained severe stress may result in significant behavioral and emotional disturbances, especially when support is not readily available [4]. It has been suggested that up to a third of military-connected children are at risk for decrements in emotional, cognitive, or physical functioning during parental deployment [8]. Two recent publications reviewed the literature on behavioral and emotional difficulties of military children 
during or after parental deployment $[9,10]$. White and colleagues reviewed nine studies (sample sizes ranging from $n=57$ to $n=642,397$ ) including two longitudinal studies and found that parental deployment was associated with increased emotional and behavioral difficulties in children [9]. Card et al. conducted a meta-analysis of 16 studies (pooled $n=19,172$ ), including three longitudinal studies, confirming this association [10]. Moreover, greater overall maladjustment, increased internalizing and externalizing problems, and poorer academic performance were the strongest during middle childhood, suggesting that children aged 6-12 years old may need more support during and after parental deployment relative to younger children and adolescents.

Prior research suggests that parental psychopathology across a range of conditions may be a risk factor for child psychopathology [11]. This suggests that elevated emotional and behavioral problems in military-connected children might stem from parental deployment-related psychopathology, given the high rates of mental health conditions such as depression and posttraumatic stress disorder (PTSD) in returning veterans. Moreover, there have been several studies examining how trauma exposure in military parents may contribute to child risk for emotional and behavioral problems [12]. For example, in a cross-sectional study of 272 military children, Lester et al. reported that parental PTSD symptoms were associated with child depression, as well as child internalizing and externalizing behaviors [13]. In this study, child internalizing behaviors were also strongly associated with parental depression and anxiety symptoms. These preliminary results need further replication and were limited by the restricted range of parental symptoms, as participants were not specifically treatment seeking.

It has been hypothesized that children are indirectly affected by the military by way of parental stress and psychopathology, with parenting factors mediating this relationship [14]. One potentially important parental factor is parenting self-efficacy or sense of competence. Prior research, predominantly cross-sectional, suggests that poor parenting self-efficacy is negatively associated with child adjustment and is positively associated with depressive symptoms and anxiety symptoms in veteran parents $[15,16]$. Thus sense of competence in parenting might be involved in the association between veteran symptoms and psychopathology in their children. However, to date, this hypothesis has not been tested. The present study aimed to examine: (1) the relationship between veteran and child psychopathology; and (2) parenting sense of competence as a mediator of the relationship between veteran psychopathology and child psychopathology.

\section{Method}

\section{Participants and Procedures}

Data for the present study were collected as part of standard clinical intake evaluations conducted at one of two community, non-VA outpatient mental health clinics treating veterans and service members: Home Base, a partnership between the Red Sox Foundation and Massachusetts General Hospital (MGH; Boston, MA) $(n=176)$ and the Road Home Program at Rush University Medical Center (Chicago, IL) $(n=39)$. Both outpatient clinics offer mental health services to veterans, service members, and their families free of charge. The combined study sample is comprised of 215 veterans and service members who reported having at least one child between the ages of 4 and 17 years and completed the Pediatric Symptom Checklist-17 (PSC17) [17]. Patients in this sample had a mean age of 37.4 years $(S D=7.9)$ and $13.3 \%(n=28)$ were female. Additional demographic characteristics are displayed in Table 1.

\section{Measures}

\section{Veteran Demographic Information}

Veterans' demographic information including age, sex, relationship status, whether or not they deployed while in the military, military rank, military branch, and military status were gathered during the intake evaluation process.

Table 1 Demographic characteristics

\begin{tabular}{lr}
\hline Characteristic & \multicolumn{1}{c}{$n(\%)$} \\
\hline Male $(n=211)$ & $183(86.7)$ \\
Married, engaged, or partnered $(n=204)$ & $123(60.3)$ \\
Deployed $(n=176)$ & $143(81.3)$ \\
Military rank $(n=190)$ & \\
Junior-level enlisted & $57(30.0)$ \\
Non-commissioned officer & $108(56.8)$ \\
Officer & $25(13.2)$ \\
Military branch $(n=205)$ & \\
Army/Army Reserve & $83(40.5)$ \\
Marines/Marine Reserve & $41(20.0)$ \\
Navy/Navy Reserve & $18(8.8)$ \\
Air Force/Air Force Reserve & $12(5.9)$ \\
National Guard & $50(24.4)$ \\
Coast Guard & $1(0.5)$ \\
Military status ( $n=204)$ & \\
Active Duty, Reserves, Inactive Ready Reserve, & $77(37.8)$ \\
$\quad$ National Guard & $127(62.3)$ \\
Discharged, Retired, Medically Retired &
\end{tabular}




\section{Veteran Measures}

Depression, Anxiety, and Stress Symptoms common to depression and anxiety were assessed using the depression anxiety stress scales (DASS-21) a 21-item self-report measure with three subscales for the respective symptom clusters [18]. Veterans were asked to rate how much each statement applied to them over the past week on a 4-point Likert scale, ranging from 0 (Never) to 3 (Almost always). Scale scores are calculated by summing the seven items in each scale such that higher ratings represent higher levels of pathology. Studies indicate that the depression (DASSD), anxiety (DASS-A), and stress (DASS-S) scales are able to distinguish well between the features of dysphoria, acute physiological arousal, and chronic tension, respectively [19]. Studies with a variety of samples, including veterans, have demonstrated that the DASS-21 has good validity and reliability $[20,21]$. Internal consistency reliability for the depression, anxiety, and stress subscales of the DASS-21 in the present study were $0.92,0.89$, and.90, respectively.

Posttraumatic Stress Disorder Home Base assessed PTSD using the PTSD Checklist-Civilian Version (PCL-C) a 17-item self-report measure assessing symptoms PTSD based on the diagnostic and statistical manual (DSM) Fourth Edition [22]. Veterans were asked to report "how much each problem has bothered them during the past week" on a 5-point Likert scale, ranging from 1 (Not at all) to 5 (Extremely). The PCL-C has been shown to have good validity and reliability in veteran samples [23, 24]. The Road Home Program assessed PTSD using the 20-item self-reported PTSD Checklist for DSM-5 (PCL5) [25]. Veterans were asked to report "how much they have been bothered by the worst event in the last month" on a 5-point Likert scale, ranging from 0 (Not at all) to 4 (Extremely). Similar to the PCL-C, the PCL-5 has been validated and shown to have good reliability in samples of veterans $[26,27]$. In order to be able to combine the two samples for the present study, the 16 common items on the PCL-C and PCL-5 were used to calculate a total score. Internal consistency for the modified version of the PCL used in this study was 0.96 , indicating good reliability of this modified measure.

Parenting Sense of Competence Veterans' sense of parental competence was assessed using the Parenting Sense of Competence Scale (PSOC) [28], a 16-item self-report assessment. Veterans are asked to report the extent to which they agree with various parenting-related statements on a 6-point Likert scale ranging from 1 (Strongly disagree) to 6 (Strongly agree). Higher scores reflect a greater sense of competence in one's parenting. The PSOC has been shown to have good validity and reliability in a variety of samples, including veterans [15]. Internal consistency for the present study was 0.86 .

\section{Child Measures Completed by the Veteran}

Pediatric Symptoms The Pediatric Symptom Checklist-17 (PSC-17) was used to assess mental health symptoms in veterans' offspring [17]. The PSC-17 is a 17-item self-report screening tool completed by children's caregivers. The PSC-17 has been found to have good validity and reliability and to provide comparable results to other well-established pediatric symptom measures [29-32]. The PSC-17 contains three subscales that capture internalizing (PSC-I), externalizing (PSC-E), and attention symptoms (PSC-A). Internalizing, externalizing, attention problems, and total symptoms are present at clinically significant levels if subscale scores are greater than or equal to $5,7,7$, and 15 , respectively [17]. At Home Base, veterans were asked to complete the measure for the child about whom they were most concerned. At the Road Home Program, veterans were asked to complete the PSC-17 on all of their children; to combine the Road Home data with the Home Base data, the most symptomatic child was identified and selected for analysis based on the highest total PSC-17 score. Internal consistency for the internalizing, externalizing, and attention subscales for the present study were $0.82,0.86,0.83$, respectively. Internal consistency for the total score was 0.91 .

\section{Procedures}

As part of their initial clinical evaluation, patients with a child between the ages of 4 and 17 completed self-report screening measures including the DASS-21, PCL-5, PSOC, and the PSC-17. Demographic and diagnostic data were routinely collected as part of the baseline clinical assessment of each patient. Clinical data were maintained in two database repositories approved by the Massachusetts General Hospital (Partners Healthcare) and the Rush University Medical Center Institutional Review Boards with a waiver of consent because all assessments were collected as part of routine clinical care procedures.

\section{Data Analyses}

Independent samples $t$-tests were conducted to test for site (MGH vs. Rush) differences in veteran psychopathology, offspring psychopathology, and parenting sense of competence. There were no significant differences on these measures by site (all $p>.24$ ). Given the similar intake evaluation processes at Home Base and the Road Home Program and the lack of statistically significant differences in any of the independent variables, mediators, or dependent variables, we combined the sample. We then conducted preliminary 
analyses to evaluate the relationship between possible demographic covariates and PSC-17 scores. Men reported that their child experienced lower symptoms on the PSC17 internalizing subscale than women did; thus, parent sex was included as a covariate in subsequent mediation analyses involving the internalizing subscale. Veteran age, relationship status, deployment status, military rank, military branch, and military status were not significantly associated with PSC-17 scores. Therefore, they were not included as covariates in mediation analyses.

Out of the 215 participants, 32 were missing scores on the modified PCL, DASS-21, or PSOC. The presence of missing data was unrelated to any of the PSC-17 variables, suggesting that the data were missing at random (MAR). Studies have shown that full-information maximum likelihood estimation (FIML) is superior to pairwise or listwise deletion under conditions of MAR [33]. Thus, we conducted FIML using Mplus version 7.3 [34] to run all analyses. Based on results from bivariate analyses, we thus tested a path analytic model examining PSOC as a mediator of the relationship between veteran psychopathology and child psychopathology. In order to determine model fit, relative $X^{2}$, root mean square error of approximation (RMSEA), comparative fit index (CFI), and standardized root mean squared residual (SRMR) were assessed. A $X^{2} / d f$ below 3.0, an RMSEA below 0.06, a SRMR below 0.08, and a CFI above 0.9 were considered to denote adequatelyfitting models [35-37]. In addition, we calculated the $90 \%$ confidence interval (CI) for the RMSEA, and the p-value for test of close fit RMSEA $\leq 0.05$.

\section{Results}

\section{Rates of Psychopathology in Children}

Based on established cutoffs for the PSC-17, 15.3\% of veterans reported having a child with significant internalizing pathology, $14.0 \%$ of veterans reported having a child with significant externalizing pathology, $18.1 \%$ of veterans reported having a child with significant attentional problems, and $30.2 \%$ of veterans reported that they had a child that met clinical threshold for at least one of the three subscales (internalizing, externalizing, attention). Based on the established cutoff for the PSC-17 total score, $20.5 \%$ of veterans reported having a child with significant overall psychopathology.

\section{Bivariate Analyses}

The estimated correlation matrix examining the relationships between veteran psychopathology, parenting sense of competence, and child psychopathology is reported
Table 2 Estimated correlation matrix of veteran psychopathology, parenting sense of competence, and child psychopathology

\begin{tabular}{lcccl}
\hline & PSC-I & PSC-E & PSC-A & PSOC \\
\hline PSOC & $-0.380^{* * *}$ & $-0.391^{* * *}$ & $-0.297^{* * *}$ & \\
Modified PCL & 0.055 & -0.005 & 0.118 & -0.105 \\
DASS-D & $0.200^{* *}$ & 0.062 & 0.126 & $-0.276^{* * *}$ \\
DASS-A & $0.135^{*}$ & 0.022 & 0.119 & $-0.218^{* *}$ \\
DASS-S & 0.130 & 0.053 & $0.208^{* *}$ & $-0.170^{*}$ \\
\hline
\end{tabular}

$N=215$. Estimated correlation matrix based full information maximum likelihood imputation in Mplus version 7.3

PSOC parenting sense of competence, Modified PCL combined PTSD Checklist score, DASS-D DASS-21 depression scale, DASS- $A$ DASS-21 anxiety scale, DASS-S DASS-21 stress scale, PSC-I Pediatric Symptom Checklist-internalizing scale, PSC-E Pediatric Symptom Checklist-externalizing scale, $P S C$ - $A$ Pediatric Symptom Checklist-attention scale

$* p<.05, * * p<.01, * * * p<.001$

in Table 2. Veterans with higher scores on the DASS21 depression and anxiety scales reported higher rates of internalizing symptoms in their children. Veterans with higher scores on the DASS-21 stress scale reported higher attentional symptoms in their children. Overall, the magnitude of these relationships was small to moderate. PTSD symptoms in veterans were not associated with child psychopathology.

There was a strong negative relationship between parenting sense of competence and all child psychopathology subscales ( $r$ from -0.294 to -0.420 , all $p<.001$ ). Higher scores on parenting sense of competence were also associated with lower scores on the DASS- 21 depression, anxiety, and stress scales ( $r$ from -0.170 to -0.276 , all $p<.05$ ). Parenting sense of competence was not associated with veteran PTSD symptoms $(r=-0.105, p=.125)$.

\section{Mediation Model}

Based on the bivariate analyses, we conducted a path analysis examining parenting sense of competence (PSOC) as a mediator of the relationship between veteran psychopathology (DASS-D, DASS-A, DASS-S) and child psychopathology (PSC-I, PSC-E, PSC-A). PTSD symptoms were not included in the model because they did not correlate with the PSOC. The structural model included direct paths from veteran psychopathology to child psychopathology as well as indirect paths via parenting sense of competence. Sex was included as a covariate by including direct paths from sex to all outcome variables. All exogenous variables were correlated with each other. We used full information maximum likelihood imputation and the MLR estimator, which is robust to non-normality. The model is depicted in Fig. 1 along with standardized path coefficients. 


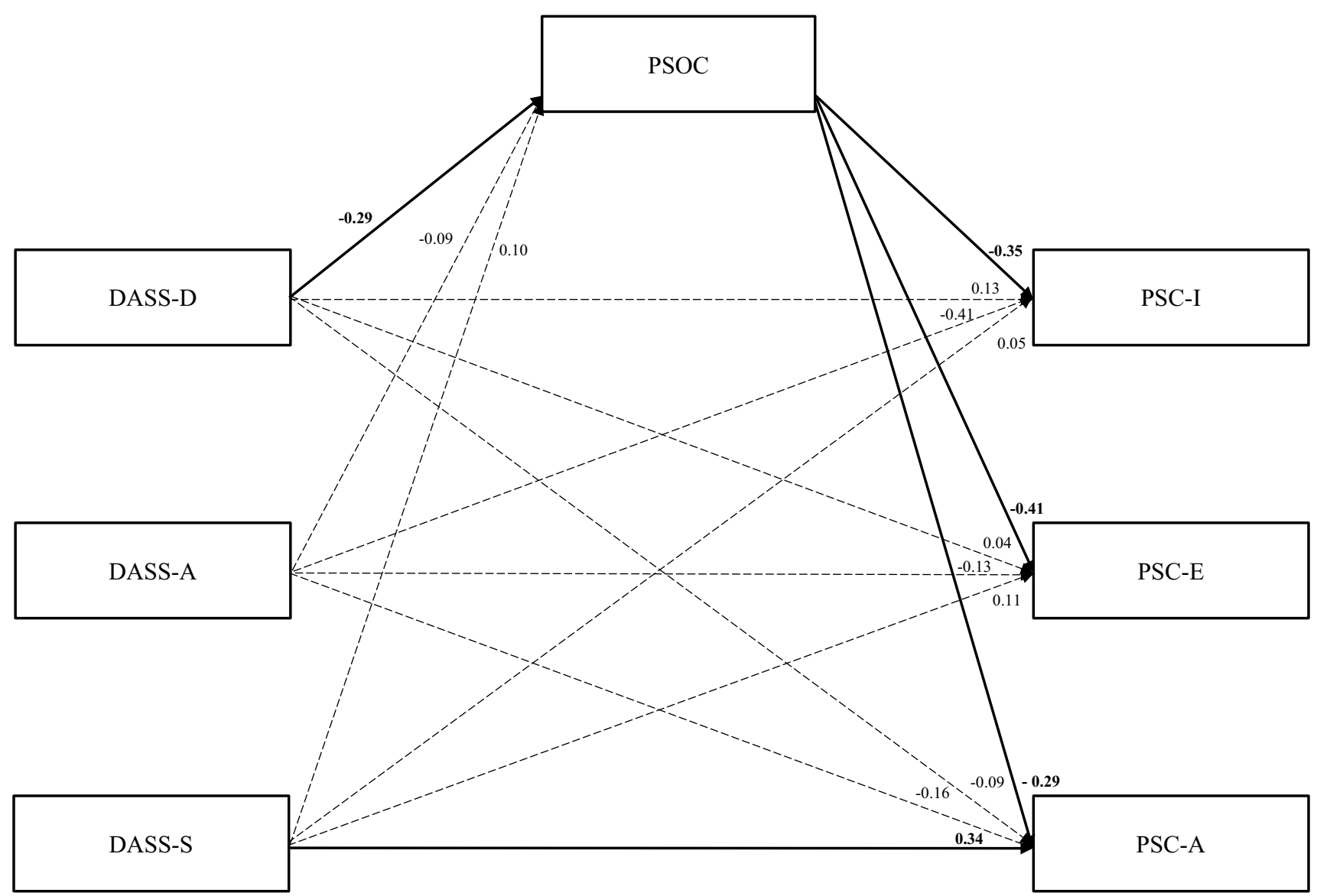

Fig. 1 Path analysis model examining parenting sense of competence as a mediator of the relationship between veteran and child psychopathol$\operatorname{ogy}\left(\chi^{2}(1)=0.384, \mathrm{p}=.54 ; \mathrm{CFI}=1.00 ; \mathrm{RMSEA}=0.000, p=.63 ; \mathrm{SRMR}=0.009\right)$

The overall model showed adequate fit to the data $\left(\chi^{2}(1)=0.384, \quad \mathrm{p}=.54 ; \quad \mathrm{CFI}=1.00 ; \quad \mathrm{RMSEA}=0.000\right.$, $p=.63$; SRMR $=0.009)$. Results showed significant indirect effects of depression (DASS-D) on all three PSC-17 outcomes via PSOC (PSC-I: Estimate $=0.019, \mathrm{SE}=0.009$, $p=.028$; PSC-E: Estimate $=0.032, \mathrm{SE}=0.013, p=.015$; PSC-A: Estimate $=0.020, \mathrm{SE}=0.009, p=.027$ ). There was also a significant direct effect of stress (DASS-S) on child attention problems (PSC-A). This model explained 14-18\% of the variance in the outcome variables. We re-ran the model without the insignificant paths to determine whether the model continued to have adequate fit once the model was less saturated (Fig. 2). The reduced model also demonstrated adequate fit to the data $\left(\chi^{2}(8)=8.840, \mathrm{p}=.36\right.$; $\mathrm{CFI}=0.996 ; \quad \mathrm{RMSEA}=0.022, \quad p=.69 ; \mathrm{SRMR}=0.029$ ). All direct paths remained significant and all three indirect paths from depression (DASS-D) to the PSC-17 outcomes via PSOC remained significant (PSC-I: Estimate $=0.021$, $\mathrm{SE}=0.008, p=.007$; PSC-E : Estimate $=0.030, \mathrm{SE}=0.009$, $p=.001$; PSC-A: Estimate $=0.017, \mathrm{SE}=0.007, p=.008$ ). This model explained $12-17 \%$ of the variance in the outcome variables.

\section{Discussion}

Rates of child psychopathology were high in our sample of treatment-seeking veterans and service members. We found almost twice the rate of children screening positive for overall psychopathology compared to a national outpatient pediatric sample $(20.5 \%$ met the cutoff for the PSC-17 total score in current sample versus $12 \%$ in national sample) [32]. These findings suggest that screening children of veterans and service members may be indicated as this would enable early identification of psychopathology in this highrisk sample. In particular, our findings suggest that veterans with higher rates of depression and anxious arousal may be more likely to have children with higher rates of internalizing symptoms. Additionally, veterans with higher rates of chronic tension may be more likely to have children with greater attentional problems. Thus, targeted screening of children may be particularly warranted in families where veterans are experiencing symptoms of depression and anxiety. Of note, the effect size of the relationships between veteran psychopathology and child psychopathology were generally small, suggesting that other factors including 
Fig. 2 Reduced path analysis model examining parenting sense of competence as a mediator of the relationship between veteran and child psychopathology $\left(\chi^{2}(8)=8.840, \mathrm{p}=.36\right.$; $\mathrm{CFI}=0.996$; $\mathrm{RMSEA}=0.022$, $p=.69 ; \mathrm{SRMR}=0.029$ )

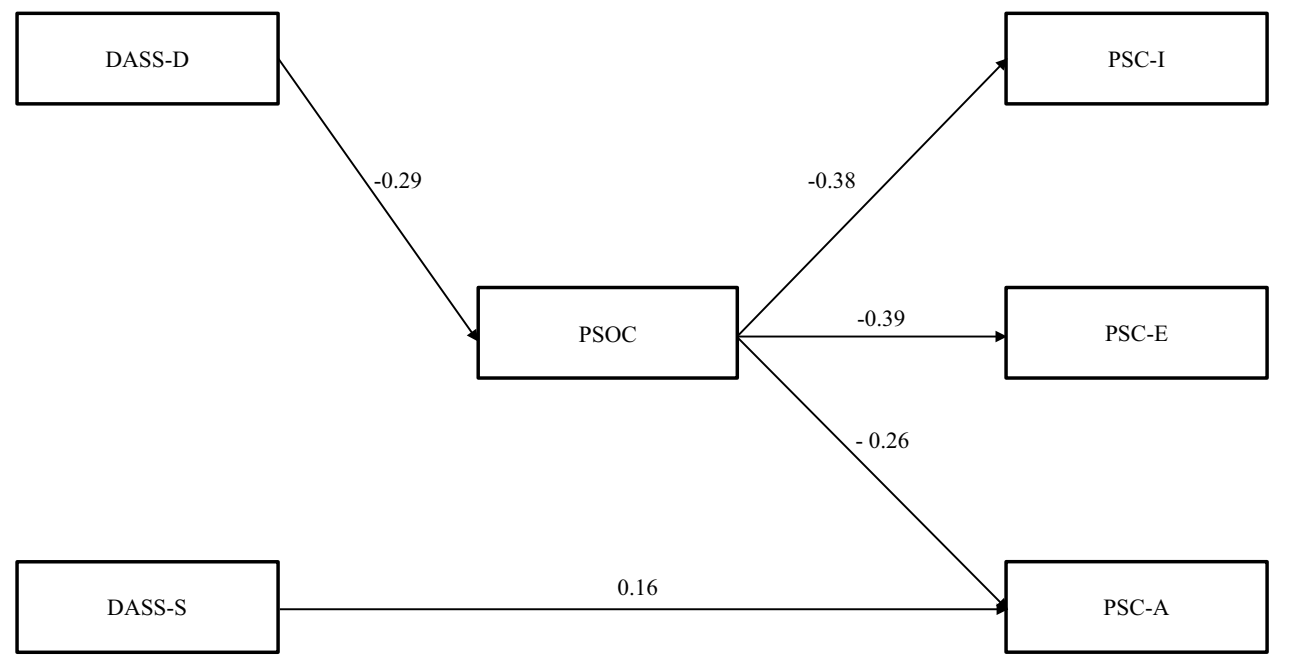

biological and genetic, as well as other environmental stressors may be implicated in the development of psychopathology in children of veterans.

Our findings provide preliminary evidence that parenting may be a critical mechanism linking veteran depression and child psychopathology. Specifically, our results show that higher depression is linked to poorer parenting sense of competence in veterans and that poorer parenting sense of competence is associated with higher rates of child internalizing, externalizing, and attentional symptoms. These findings are based on cross-sectional data and thus do not inform the direction of these relationships. Our findings, however, provide preliminary evidence for the hypothesis that enhancing parenting sense of competence among veterans with depression may be a useful intervention target for reducing psychopathology in military-connected children; research examining the effect of this type of parenting intervention with military families is needed. Prior research indicates that parenting interventions can increase parental sense of competence [38]; thus parenting self-efficacy appears to be a modifiable target.

There are several reasons why parents who are struggling with depression may feel less capable of caring for their children. First, negative biases about the self that maintain depression are likely to carry over into self-perceptions of parenting. Additionally, symptoms of depression and resulting functional impairment may prevent veteran parents from interacting with children in ways that boost confidence. For example, parents with depression have been shown to exhibit less positive affect and more negative affect during observed interactions with their children [39]. Parents with depression may also find it more difficult to manage negative interactions with their children. For example, one study showed that depressed parents are more likely to use punitive disciplinary behaviors and are less likely to use some positive disciplinary strategies [40].
Understanding how depression is linked to poorer sense of competence in parenting would be valuable for identifying possible targets for intervention in this population.

Our study also revealed several ways in which veteran psychopathology and child psychopathology were not linked. Externalizing symptoms in children were not associated with any type of veteran psychopathology even though parenting sense of competence was linked to externalizing symptoms. This may suggest that for parents of children with externalizing problems, poor parenting sense of competence may stem from the challenges that come with dealing with the child's difficulties rather than one's own psychopathology; however we did not evaluate externalizing forms of veteran psychopathology in this study, so further research is needed. Moreover, veteran PTSD symptoms did not appear to be associated with child psychopathology or parenting sense of competence in this sample. These findings are inconsistent with previous research [13] and are particularly striking given that PTSD is often a primary focus in veteran samples. It is possible that differences may be due to our modified PCL, which combined different versions of the PCL that asked about different timeframes, though this is unlikely given the high internal reliability of our modified measure. Further research is needed to explore potential moderating factors that may explain these inconsistent results.

Several limitations should be taken into consideration when interpreting the results of this study. Our results are cross-sectional and relied exclusively on parental selfreport. This means that directional interpretations cannot be made. It is possible that child psychopathology contributes to a diminished sense of parental competence, which in turns leads to parental depression. Moreover, it is possible that parental ratings of their children's psychopathology could be influenced by their own mood states [30]. Longitudinal research is needed using multiple informants to 
explore causal links between parental psychopathology and child psychopathology in military families. Because we had no demographic information about the children, we were unable to determine whether child sex or age influenced the observed relationships. We also did not explore the co-parenting relationship between veterans and their partners or partner psychopathology. It is possible that veteran psychopathology may be associated with discord between the partners, which influences parenting and child psychopathology. It is also plausible that partner psychopathology may interact with veteran psychopathology in determining child outcomes. For example, having a partner with low psychopathology may serve as a protective buffer for children. Finally, we did not have a non-military control group; thus, it is impossible to establish the degree to which the observed results are driven by military experiences.

Despite these limitations, this study highlights the association between parental psychopathology and children's mental health in military families. Moreover, the identification of the role of parenting competence in this relationship represents an important step towards understanding mechanisms that link veteran and child psychopathology. It is clear that military children represent an important high-risk group and that further quantitative and qualitative research is needed to understand the factors that enhance vulnerability and that can serve as potential targets for intervention.

\section{Summary}

In this study, we evaluated the relationship between parent and child psychopathology in treatment-seeking veterans and explored parental sense of competence as a potential mediator of this relationship. We found high rates of child psychopathology in our sample, indicating that further attention is needed to screen for and address mental health concerns in this population. In particular, our findings suggest that veterans with higher rates of depression and anxious arousal may be more likely to have children with higher rates of internalizing symptoms and that veterans with higher rates of chronic tension may be more likely to have children with greater attentional problems. Parenting sense of competence significantly mediated the relationship between veteran depression and child psychopathology, suggesting that for veterans with depression and poor parenting sense of competence, interventions that aim to build parenting self-efficacy warrant further investigation as a strategy for enhancing child well being.

Acknowledgements General program support for the Road Home Program: Center for Veterans \& their Families comes from core grants from Wounded Warrior Project; Welcome Back Veterans, an initiative of the Robert R. McCormick Foundation and Major League Baseball; and The Crown Family. The Children \& Families Program at Road Home Program is supported by a grant from the Michael Reese Health Trust. Home Base is a philanthropic effort of the Red Sox Foundation and Massachusetts General Hospital. Drs. Bui, Ohye, Rauch, and Simon's research efforts are supported by Home Base. Alyson Zalta receives grant support from the National Institute of Mental Health (K23 MH103394).

\section{Compliance with Ethical Standards}

Conflict of interest Eric Bui, Lauren Laifer, Julia Sager, Denise Zou, and Bonnie Ohye have no conflicts of interest to report. Alyson Zalta receives grant support from the National Institute of Mental Health and the Brain \& Behavior Foundation. Dr. Zalta's spouse serves on the Clinical Advisory Committee for Ginger.io, serves on the Scientific Advisory Committee for Kemoko, Inc., and owns equity in Optilife Technologies, Inc. Philip Held receives grant support from the Cohn Family Foundation. Paula Rauch receives author royalties from McGraw-Hill Publishing. Naomi Simon receives grant support from the American Foundation for Suicide Prevention, Department of Defense, Highland Street Foundation, National Institute of Health, and Massachusetts Attorney General's Office. Dr. Simon has been reimbursed by the MGH Psychiatry Academy for discussing and presenting research at meetings, conferences, and symposiums. Dr. Simon's spouse owns equity in G1 Therapeutics and Gatekeeper. Dr. Karnik serves on the Board of Directors of the Generations of Hope Developmental Corporation. Dr. Karnik presently has grant support from the Substance Abuse Mental Health Services Administration, National Institutes of Health, Wounded Warrior Project, Bob Woodruff Foundation, Tawani Foundation, and the Michael Reese Health Trust. Dr. Pollack receives grant support from the National Institute of Health, Janssen, and Edgemont; he is on the advisory board/provides consultation to Clintara, Edgemont Pharmaceuticals, and Palo Alto Health Sciences; he owns equity in Doyen Medical, Medavante, Mensante Corportaion, Mindsite, and Targia Pharmaceuticals; he receives royalties from the SIGH-A, and SAFER interviews.

Open Access This article is distributed under the terms of the Creative Commons Attribution 4.0 International License (http:// creativecommons.org/licenses/by/4.0/), which permits unrestricted use, distribution, and reproduction in any medium, provided you give appropriate credit to the original author(s) and the source, provide a link to the Creative Commons license, and indicate if changes were made.

\section{References}

1. National Center for Veterans Analysis and Statistics (2016) Profile of post-9/11 veterans: 2014. Retrieved from https://www. va.gov/vetdata/docs/SpecialReports/Post_911_Veterans_Profile_2014.pdf

2. Tanielian TL, Jaycox L (2008) Invisible wounds of war : psychological and cognitive injuries, their consequences, and services to assist recovery. RAND, Santa Monica

3. Department of Defense (2010) Report on the impact of deployment of members of the armed forces on their dependent children

4. Esposito-Smythers C, Wolff J, Lemmon KM, Bodzy M, Swenson RR, Spirito A (2011) Military youth and the deployment cycle: emotional health consequences and recommendations for intervention. J Fam Psychol 25(4):497-507

5. Huebner AJ, Mancini JA (2005) Adjustments among adolescents in military families when a parent is deployed. Final report to the Military Family Research Institute and Department of Defense Quality of Life Office. Retrieved from http://www. 
juvenilecouncil.gov/materials/june_8_2007/MFRI\%20final\%20 report\%20JUNE\%202005.pdf. Accessed 12 April 2017

6. Chandra A, Lara-Cinisomo S, Jaycox LH, Tanielian T, Han B, Burns RM, Ruder T (2011) Views from the homefront: the experiences of youth and spouses from military families. Retrieved from http://www.rand.org/pubs/periodicals/health-quarterly/ issues/v1/n1/12.html. Accessed 12 April 2017

7. Compas BE, Banez GA, Malcarne VL (1992) Coping with psychosocial stress: a developmental perspective. In: Carpenter B (ed) Personal coping: theory, research, and its application. Pergamon, New York

8. Flake EM, Davis BE, Johnson PL, Middleton LS (2009) The psychosocial effects of deployment on military children. J Dev Behav Pediatr 30(4):271-278

9. White CJ, de Burgh HT, Fear NT, Iversen AC (2011) The impact of deployment to Iraq or Afghanistan on military children: a review of the literature. Int Rev Psychiatry 23(2):210-217

10. Card NA, Bosch L, Casper DM, Wiggs CB, Hawkins SA, Schlomer GL, Borden LM (2011) A meta-analytic review of internalizing, externalizing, and academic adjustment among children of deployed military service members. J Fam Psychol 25(4):508-520

11. Goodman SH, Gotlib IH (1999) Risk for psychopathology in the children of depressed mothers: A developmental model for understanding mechanisms of transmission. Psychol Rev 106(3):458-490

12. Dekel R, Goldblatt H (2008) Is there intergenerational transmission of trauma? The case of combat Veterans' children. Am J Orthopsychiatr 78:281-289

13. Lester P, Peterson K, Reeves J et al (2010) The long war and parental combat deployment: Effects on military children and at-home spouses. J Am Acad Child Adolesc Psychiatry 49(4):310-320

14. Palmer C (2008) A theory of risk and resilience factors in military families. Mil Psychol 20(3):205-217

15. Bui E, Zakarian RJ, Laifer LM et al (2017) Psychometric properties of the parenting sense of competence scale in treatmentseeking post-9/11 veterans. J Child Fam Stud 26:464-470

16. Jones TL, Prinz RJ (2005) Potential roles of parental self-efficacy in parent and child adjustment: a review. Clin Psychol Rev 25(3):341-363

17. Gardner W, Murphy JM, Childs G et al (1999) The PSC-17: A brief pediatric symptom checklist with psychosocial problem subscales. a report from PROS and ASPN. Ambul Child Health 5:225-236

18. Lovibond PF, Lovibond SH (1995) The structure of negative emotional states: comparison of the depression anxiety stress scales (DASS) with the beck depression and anxiety inventories. Behav Res Ther 33:335-343

19. Antony MM, Bieling PJ, Cox BJ, Enns MW, Swinson RP (1998) Psychometric properties of the 42-item and 21-item versions of the depression anxiety stress scales in clinical groups and a community sample. Psychol Assess 10(2):176-181

20. Brown TA, Chorpita BF, Korotitsch W, Barlow DH (1997) Psychometric properties of the depression anxiety stress scales (DASS) in clinical samples. Behav Res Ther 35(1):79-89

21. Osman A, Wong JL, Bagge CL, Freedenthal S, Gutierrez PM, Lozano G (2012) The Depression Anxiety Stress Scales-21 (DASS-21): further examination of dimensions, scale reliability, and correlates. J Clin Psychol 68(12):1322-1338

22. Weathers F, Litz B, Herman D, Huska J, Keane T (1993) The PTSD Checklist (PCL): reliability, validity, and diagnostic utility. Annual Convention of the International Society for Traumatic Stress Studies, San Antonio
23. Searle AK, Van Hooff M, McFarlane AC et al (2015) The validity of military screening for mental health problems: diagnostic accuracy of the PCL, K10 and AUDIT scales in an entire military population. Int J Methods Psychiatr Res 24(1):32-45

24. Dobie DJ, Kivlahan DR, Maynard C, Bush KR, McFall M, Epler AJ, Bradley KA (2002) Screening for post-traumatic stress disorder in female Veteran's Affairs patients: Validation of the PTSD checklist. Gen Hosp Psychiatry 24(6):367-374

25. Weathers FW, Litz BT, Keane TM, Palmieri PA, Marx BP, Schnurr PP (2013) The PTSD Checklist for DSM-5 (PCL-5). Scale available from the National Center for PTSD. www.ptsd. va.gov

26. Bovin MJ, Marx BP, Weathers FW, Gallagher MW, Rodriguez P, Schnurr PP, Keane TM (2016) Psychometric properties of the PTSD checklist for diagnostic and statistical manual of mental disorders-fifth edition (PCL-5) in veterans. Psychol Assess 28(11):1379-1391

27. Wortmann JH, Jordan AH, Weathers FW et al (2016) Psychometric analysis of the PTSD Checklist-5 (PCL-5) among treatment-seeking military service members. Psychol. Assessment 28(11):1392-1403

28. Johnston C, Mash EJ (1989) A measure of parenting satisfaction and efficacy. J Clin Child Psychol 18:167-175

29. Gardner W, Lucas A, Kolko DJ, Campo JV (2007) Comparison of the PSC-17 and alternative mental health screens in an atrisk primary care sample. J Am Acad Child Adolesc Psychiatry 46(5):611-618

30. Borowsky IW, Mozayeny S, Ireland M (2003) Brief psychosocial screening at health supervision and acute care visits. Pediatrics 112(1):129-133

31. Duke N, Ireland M, Borowsky IW (2005) Identifying psychosocial problems among youth: Factors associated with youth agreement on a positive parent-completed PSC-17. Child Care Health Dev 31(5):563-573

32. Murphy JM, Bergmann P, Chiang C, Sturner R, Howard B, Abel MR, Jellinek M (2016) The PSC-17: subscale scores, reliability, and factor structure in a new national sample. Pediatrics. doi:10.1542/peds.2016-0038

33. Muthén B, Kaplan D, Hollis M (1987) On structural equation modeling with data that are not missing completely at random. Psychometrika 52(3):431-462

34. Muthén LK, Muthén BO (2015) Mplus user's guide, 7th edn. Muthén \& Muthén, Los Angeles

35. Hu L, Bentler PM (1999) Cutoff criteria for fit indexes in covariance structure analysis: conventional criteria versus new alternatives. Struct Equ Model 6(1):1-55

36. Browne MW, Cudeck R (1993) Alternative ways of assessing model fit. In: Bollen KA, Long JS (eds) Testing structural equation models. Sage, Newbury Park, pp 136-162

37. Kline RB (2015) Principles and practice of structural equation modeling. Guilford, New York

38. Stattin H, Enebrink P, Ozdemir M, Giannotta F (2015) A national evaluation of parenting programs in Sweden: the shortterm effects using an RCT effectiveness design. J Consult Clin Psychol 83(6):1069-1084

39. Muzik M, Morelen D, Hruschak J, Rosenblum KL, Bocknek E, Beeghly M (2017) Psychopathology and parenting: an examination of perceived and observed parenting in mothers with depression and PTSD. J Affect Disord 207:242-250

40. Davis DW, Myers J, Logsdon MC, Bauer NS (2016) The relationship among caregiver depressive symptoms, parenting behavior, and family-centered care. J Pediatr Health Care 30(2):121-132 